\title{
CALIDAD DEL AGUA DEL REFUGIO MATA REDONDA Y LOS ARROZALES COLINDANTES, GUANACASTE, COSTA RICA ${ }^{1}$
}

\author{
Ana Gabriela Pérez-Castillo², Ronny Barboza-Mora3 ${ }^{3}$,José Francisco Ramos-Matarrita ${ }^{4}$
}

\begin{abstract}
RESUMEN
Calidad del agua del Refugio Mata Redonda y los arrozales colindantes, Guanacaste, Costa Rica. El objetivo de este trabajo fue evaluar si el agua de la laguna Mata Redonda (LMR) y de la actividad arrocera en el sector San Lázaro (SSL) de Nicoya, es apropiada para el sostenimiento de la biodiversidad y el desarrollo de la vida acuática. El análisis de la calidad fisicoquímica del agua se llevó a cabo mediante el índice de calidad de agua para el manejo de lagunas de inundación (ICA-L) a partir de datos de temperatura, $\mathrm{pH}$, porcentaje de saturación de oxígeno, conductividad eléctrica, demanda bioquímica de oxígeno, sólidos suspendidos, nitratos y fósforo total. El ICA-L promedio del agua que drenó de los arrozales por el canal principal, (ICA-Lp) fue 42 , correspondiente a la categoría "mala" (no apta para el sostenimiento de la biodiversidad de lagunas de inundación). En la LMR (ICA-Lp 80) y en la salida de la laguna (ICA-Lp 81) la calidad del agua se clasificó como "buena". No obstante incrementos en la concentración de fósforo total y en la demanda biológica de oxígeno (DBO) conducirían a un deterioro del ecosistema. El nivel de riesgo de los plaguicidas aplicados a los arrozales durante el período en estudio, bajo las condiciones del SSL, fue estimado mediante el Pesticide Index Rating Impact (PIRI); los resultados mostraron que la deltametrina, esfenvalerato, permetrina y butaclor, por su alta toxicidad y movilidad, serían los menos apropiados de aplicar. En tanto que, imidapropid, bispiribac-Na, triazofos, glifosato, acefato, clomazone y tricopyr, resultarían poco peligrosos bajo prácticas de aplicación apropiadas.
\end{abstract}

Palabras clave: calidad del agua, arroz, plaguicidas en arrozales, plaguicidas en humedales.

\begin{abstract}
Water quality of the Refugio Mata Redonda and surrounding rice paddies, Guanacaste, Costa Rica. The objective of this study was assess if the water quality from the Mata Redonda lagoon (MRL) and from the rice fields in San Lazaro sector of Nicoya, is appropriate for biodiversity and aquatic life development. The water quality analysis was carried out using the Water quality index for managing flood lagoons (ICA-L), which is based on the following data: temperature, $\mathrm{pH}$, oxygen saturation percentage, electrical conductivity, biochemical oxygen demand, suspended solids, nitrate content and total phosphorus content. The ICA-L average (ICA-Lp) from the water drained form the rice fields in the main channel was 42 , which corresponds to a "bad" category value (unfit for sustaining biodiversity in flood lagoons). In the MRL (ICA-Lp 80) and in the lagoon's drainage (ICA-Lp 81), water quality was classified as "good". Nevertheless, increases in total phosphorus and the biological oxygen demand (BOD) may cause negative changes in the composition of the ecosystem. The risk level associated with pesticide application in rice fields (under the San Lazaro area conditions during the study period) was estimated using the Pesticide Impact Rating Index (PIRI). The PIRI results showed that the less appropriate to use pesticides are: deltamethrin, esfenvalerate, permethrin and butachlor, because of their high toxicity and mobility. On the other hand, imidapropid, bispyribac-Na, triazophos, glyphosate, acephate, clomazone and tricopyr, would be less dangerous if applied under appropriate practices.
\end{abstract}

Keywords: water quality, rice,pesticides, agro-chemicals in wetlands.

\footnotetext{
1 Recibido: 19 de setiembre, 2012. Aceptado: 28 de octubre, 2013. Contiene parte de los datos de la tesis del tercer autor.

2 Centro de Investigaciones en Contaminación Ambiental, Universidad de Costa Rica. San José, Costa Rica. Apdo. postal 2060 Ciudad Universitaria Rodrigo Facio. ana.perezcastillo@ucr.ac.cr

3 Centro de Investigaciones en Granos y Semillas (CIGRAS), Universidad de Costa Rica. San José, Costa Rica. ronny.barboza@ucr.ac.cr

4 Sistema Nacional de Áreas de Conservación, SINAC-CIPANCI. San José, Costa Rica. fcoramosm@gmail.com
} 


\section{INTRODUCCIÓN}

El Refugio Nacional de Vida Silvestre Mata Redonda (RNVS-MR) del Sistema Nacional de Áreas de Conservación (SINAC) de Costa Rica (coordenadas CRTM05 380553 y 256933), está ubicado en la margen derecha del río Tempisque a lo largo de la cuenca baja, entre la desembocadura del río El Charco y la comunidad de Rosario, en el cantón de Nicoya, provincia de Guanacaste.

El RNVS-MR es un importante humedal palustre, que tiene alta biodiversidad y es parte del corredor biológico que comprende los humedales Corral de Piedra, Las Delicias, La Bolsa, La Jacinta, así como, los humedales del Parque Nacional Palo Verde (PNPV). Dichos humedales se caracterizan por la relativa cercanía entre ellos y por un proceso de secamiento natural diferenciado conforme avanza la época seca. Esta situación, permite un balance en la disponibilidad de alimento durante la época seca entre los diferentes humedales, que condiciona la migración de las aves entre un sitio y otro (Piedra 2005).

Los cultivos de arroz se ubican al suroeste del RNVS-MR en el sector de San Lázaro (SSL) y conforman parte de la zona de amortiguamiento del RNVSMR, de 2402 ha, propuesta por el Área de Conservación Tempisque del SINAC, entidad que ha estimado que las actividades desarrolladas como parte de estos ciclos de cultivo influencian directamente el Refugio de 372 ha (Valverde 2006).

La actividad arrocera se desarrolla en el entorno de la Laguna Mata Redonda (LMR) debido a la predominancia de suelos vertisoles, aptos para este cultivo por su alta capacidad de retención de humedad; tradicionalmente se incluyen las fincas Los Montecillos con 650 ha y La Cueva con 500 ha que son terrenos pantanosos donde el nivel freático es mínimo, que se secan rápidamente al iniciar la época seca y tienen poblados de plantas acuáticas, algunas leñosas y pastos naturales 5 .

Las dos fincas son atravesadas por el río San Lázaro, el cual alcanza un caudal de $2,25 \mathrm{~m}^{3} / \mathrm{s}$ durante la época lluviosa y disminuye hasta casi desaparecer en la época seca. Esto restringe la producción en los años secos a una sola cosecha, que depende

\footnotetext{
Delgado, V. 2009. Tamaño de fincas arroceras y características de los suelos para el cultivo de arroz en San Lázaro, Nicoya, Guanacaste. Comunicación personal.
}

mayoritariamente de la precipitación de la época lluviosa y del riego suplementario para reducir el déficit hídrico ${ }^{6}$.

La mayor parte de estos suelos, en su condición original, fueron humedales que se trasformaron paulatinamente mediante drenajes, construcción de terrazas, muros de contención entre otros, para brindar las condiciones técnicas requeridas por el cultivo del arroz. Inicialmente se cultivaron parcelas pequeñas destinadas al autoconsumo, pero a partir de la década de los 70’s se inició la siembra de extensas áreas bajo la modalidad de secano y luego de 1990, se transformó el sistema productivo a secano favorecido que incluye riego suplementario por inundación (Jiménez y González 2001, 2003).

Con la desaparición de los humedales naturales, las aves acuáticas se han desplazado hacia los arrozales que se hallan bajo una lámina de agua. Según Hurtado (2003), su distribución entre ambos sistemas se explica por la profundidad del agua, los componentes de cobertura y la disponibilidad o accesibilidad a las presas.

Las posibles repercusiones ambientales negativas del cultivo del arroz pueden originarse por el cambio de uso del suelo, la utilización de plaguicidas tóxicos y móviles, en especial si se utiliza aspersión con avioneta, y el arrastre de contaminantes y sólidos suspendidos desde los drenajes hacia aguas superficiales. Al drenar el agua de las terrazas, por ejemplo antes de la cosecha, o por mal manejo del agua en sistemas de inundación, la corriente podría arrastrar residuos de los fertilizantes y de los plaguicidas aplicados en el proceso a fuentes de agua superficial (Rizo-Patrón 2003).

Estudios previos de las aguas de drenaje de arrozales de los sectores de Bagatsí y Tamarindo del Distrito de Riego Arenal-Tempisque, han demostrado la presencia de plaguicidas, grasas, aceites y otros contaminantes; así como variaciones significativas en la calidad de las aguas, por las descargas de sales y fertilizantes que incidieron en la concentración de fósforo total, la acidez y la conductividad (Rodríguez 1996, Rizo-Patrón, 2003, Pérez 2010).

El balance hídrico de la LMR, el seguimiento periódico del estado de la biodiversidad y la determinación de la calidad del agua en época lluviosa y seca,

\footnotetext{
Delgado, V. 2009. Caudal del río San Lázaro y posibilidades de segunda cosecha de arroz en San Lázaro, Nicoya, Guanacaste. Comunicación personal.
} 
son aspectos relevantes pendientes de estudio. Aunque el RNVS-MR colinda con cultivos de arroz cuyos drenajes vierten hacia la LMR, no se han realizado investigaciones para evaluar el efecto ambiental de la actividad arrocera sobre el RNVS-MR. El objetivo de este trabajo fue evaluar si el agua de la laguna Mata Redonda (LMR) y de la actividad arrocera en el sector San Lázaro (SSL) de Nicoya, es apropiada para el sostenimiento de la biodiversidad y el desarrollo de la vida acuática.

\section{MATERIALES Y MÉTODOS}

\section{Recolección de información de la actividad arrocera}

La información sobre el área de siembra en la temporada agrícola, las prácticas agronómicas utilizadas, el sistema de combate de plagas y los productos utilizados se recopiló en junio de 2009, mediante una entrevista estructurada al Ing. Víctor Delgado A, responsable de la producción arrocera en el SSL durante el ciclo productivo en estudio.

Las prácticas agronómicas y la tecnología del cultivo de arroz utilizada en el SSL, fueron verificadas por medio de visitas semanales a la plantación de arroz. Se caracterizó la infraestructura de riego, la preparación de suelos, el sistema de siembra y las variedades utilizadas, el programa de fertilización, los programas de combate de arvenses y de plagas, los métodos de aplicación de plaguicidas, el sistema de cosecha y el manejo de residuos tanto de envases de agroquímicos como de cosecha.

\section{Plan de muestreo de aguas}

Entre el 15 de julio y el 30 de noviembre del 2009, se realizaron giras de muestreo, aproximadamente cada mes, y se tomaron muestras simples de agua para los diversos análisis, en los siguientes sitios:

- Salida del drenaje principal del SSL (sitio 1). Coordenadas CRTM05 0378203 y 0254876 , ubicado en la esquina noreste del cultivo de arroz.

- Zona central de la LMR (sitio 2). Coordenadas CRTM05 0380553 y 0256933.

- Efluente principal de LMR (sitio 3). Coordenadas CRTM 050381270 y 0257537 . La sedimentación del desagüe de la laguna originó la apertura de cauces de drenaje secundarios.

\section{Toma de muestras}

Las muestras se tomaron conforme al procedimiento acreditado por el Centro de Investigación en Contaminación Ambiental (CICA), sustentando en apartado 1060 del Standard Methods for the Examination of Water and Wastewater de la American Water Works Association (Eaton et al. 2005).

Los recipientes, se enjuagaron tres veces con el agua del sitio y, según la variable a cuantificar, se procedió conforme a las condiciones que se especifican a continuación:

Fósforo total: botellas de vidrio color ámbar, enjuagadas con detergente sin fosfatos, $\mathrm{HCl}$ diluido caliente y finalmente, agua destilada. Las muestras se preservaron con $1 \mathrm{ml}$ de $\mathrm{HCl}$ concentrado por litro.

Demanda biológica de oxígeno: botellas de polietileno color ámbar. Su análisis se realizó antes de 36 horas de transcurrido el muestreo.

Nitrato: botellas de polipropileno blancas. Se empleó como agente preservante $4 \mathrm{ml} / \mathrm{l}$ de cloroformo.

Sólidos suspendidos: botellas de polietileno transparentes.

Plaguicidas: botellas de vidrio ámbar de un litro, enjuagadas previamente con agua desionizada y acetona. Las muestras para analizar plaguicidas ácidos, se conservaron con sulfito de sodio y las correspondientes para la determinación de carbamatos, se llevaron a un $\mathrm{pH}$ ácido.

La toma de muestras para la determinación de plaguicidas se restringió a los sitios 1 y 2 .

Las muestras se conservaron en hielo protegidas con bolsas plásticas cerradas mediante un nudo desde su recolección hasta su ingreso al laboratorio. Para disminuir su descomposición microbiológica, aparte de aplicar el preservante indicado según la variable a estudiar, se mantuvieron a $4^{\circ} \mathrm{C}$ hasta el día del análisis, momento en que se llevaron a temperatura ambiente.

\section{Calidad fisicoquímica del agua}

Se midieron en el sitio de muestreo mediante equipo calibrado y verificado previamente las siguientes variables:

La temperatura con un termómetro de vidrio con resolución de $\pm 0,1^{\circ} \mathrm{C}$, la conductividad, el oxígeno disuelto y el porcentaje de saturación de oxígeno, con un medidor multiparámetro, y el $\mathrm{pH}$ con un $\mathrm{pH}$-metro.

La concentración de fósforo total, de nitrato, de sólidos suspendidos y la demanda biológica de oxígeno 
para cinco días, se determinaron en el Laboratorio de Calidad de Aguas del CICA, bajo métodos de análisis validados y acreditados por dicho Centro, conforme a metodologías fundamentadas en el Standard Methods for the Examination of Water and Wastewater (Eaton et al. 2005).

Para la cuantificación de fósforo total, la muestra se digirió con $\mathrm{HNO}_{3}$ en un microondas y luego fue medida por el método de cloruro estañoso, utilizando un espectrofotómetro UV-visible a $690 \mathrm{~nm}$ según apartado 4500-P-D (Eaton et al. 2005). La concentración de nitrato fue determinada por cromatografía líquida de intercambio iónico y detector de conductividad con supresión química de la conductividad del efluente, según el apartado 4110-B (Eaton et al. 2005), haciendo uso de una columna aniónica $100 / 4,0 \mathrm{~mm}$ y una fase móvil 3,2 mmol de $\mathrm{Na}_{2} \mathrm{CO}_{3}$ y 1,0 mmol de $\mathrm{NaHCO}_{3}$.

\section{Trazas de plaguicidas}

La cuantificación de 44 plaguicidas en el agua se llevó a cabo según los procedimientos de análisis MAR-01, análisis de multirresiduos por cromatografía de gases y MAR-07, determinación de plaguicidas ácidos y básicos por cromatografía líquida, vigentes, validados y acreditados por el Laboratorio de Plaguicidas del CICA. La preparación de la muestra bajo estas técnicas se sustentó, respectivamente, en la sección 302 del Pesticide Analytical Manual (FDA 1999) y en el método propuesto por Di Corcia y Marchetti (1992) y Di Corcia y Samperi (1993).

La cuantificación en MAR-01 se llevó a cabo con un cromatógrafo de gases y detección en modo DUAL con microcaptura electrónica $(\mu$-ECD) y fotometría de llama (FPD). Se emplean columnas de 30 $\mathrm{m} \times 0,25 \mathrm{~mm} \times 0,25 \mu \mathrm{m}$, con una fase estacionaria de 95\% metil polisiloxano y $5 \%$ fenil metil siloxano. La temperatura del horno inició a $50^{\circ} \mathrm{C}$ con una rampa de $25^{\circ} \mathrm{C} /$ min hasta $200^{\circ} \mathrm{C}$, luego pasó de $200^{\circ} \mathrm{C}$ a $275^{\circ} \mathrm{C}$ a razón de $2^{\circ} \mathrm{C} / \mathrm{min}$ y de $275^{\circ} \mathrm{C}$ a $290^{\circ} \mathrm{C}$ a razón de $25^{\circ} \mathrm{C} / \mathrm{min}$, temperatura final en la cual permanecieron por dos minutos. El tiempo total de cada corrida cromatográfica fue de 46,1 min. Otras condiciones cromatográficas son las siguientes: gas de arrastre $\mathrm{N}_{2}$ ultrapuro $(99,999 \%)$, pasado a través de una trampa molecular para humedad y otra para oxígeno con un flujo constante de $1,1 \mathrm{ml} / \mathrm{min}$ para una velocidad lineal de $28 \mathrm{~cm} / \mathrm{s}$. Temperatura del puerto de inyección $275^{\circ} \mathrm{C}$, usado con una razón de inyección automática en modo splitless. Temperatura del detector $\mu$-ECD $300^{\circ} \mathrm{C}$ constante, con gas $\mathrm{N}_{2}$ como make up a un flujo de $40 \mathrm{ml} / \mathrm{min}$. Detector FPD que recibe hidrógeno a un flujo de $75 \mathrm{ml} / \mathrm{min}$ con una pureza de $99,999 \%$ y aire cero a razón de $100 \mathrm{ml} / \mathrm{min}$, además de $\mathrm{N}_{2}$ como make up a razón de $15 \mathrm{ml} / \mathrm{min}$. El análisis confirmatorio se realizó con un cromatógrafo de gases acoplado a un detector de espectrómetro de masas que utiliza $\mathrm{He}$ de 99,999\%. Las condiciones cromatográficas se repiten excepto por la temperatura del detector de masas, donde la fuente de ionización y el cuadrupolo se programaron a $230^{\circ} \mathrm{C}$ y a $150^{\circ} \mathrm{C}$, respectivamente.

La cuantificación con el método MAR-07 se realizó con un cromatógrafo líquido de alta resolución y detector de arreglo de diodos G1315C operado a $1220 \mathrm{~nm}$ y $254 \mathrm{~nm}$. Se empleó una columna C-18 de $5 \mu \mathrm{m}$ x 4,6 $\mathrm{mm}$ x $25 \mathrm{~cm}$. La fase móvil a un flujo de $1,00 \mathrm{ml} / \mathrm{min}$, se conformó de una mezcla acetonitrilo: amortiguador de fosfato (concentración de $13 \mathrm{mmol} / \mathrm{l}$ y pH 3,40 \pm $0,02)$. El gradiente aplicado fue del tiempo cero al minuto diez, aumento en la proporción de acetonitrilo de $15 \%$ a $45 \%$, fase móvil constante por siete minutos, luego del minuto 17 al 20 incremento del acetonitrilo de $45 \%$ a $70 \%$ y de nuevo fase móvil constante por diez minutos. Finalmente, el acetonitrilo cae de $70 \%$ a la condición inicial de $15 \%$ en un minuto. Del minuto 31 al 37 se regenera la columna.

Las incertidumbres asociadas a los resultados de los análisis, se sustentaron a partir de la validación de los métodos de ensayo realizados por el Laboratorio de Calidad de Aguas y el Laboratorio de Análisis de Plaguicidas del CICA, estudios que incorporaron el cálculo de la variabilidad y la incertidumbre estándar de los datos.

\section{Evaluación de la calidad del agua}

Para la evaluación de la calidad del agua se empleó el Índice de Calidad de Agua para lagunas de inundación (ICA-L) (Pérez 2008), tanto en el RNVS-MR como en los canales de drenaje del cultivo de arroz en el SSL, bajo escala de comparación (Cuadro 1).

\section{Evaluación del riesgo de plaguicidas para ecosiste- mas de humedal}

De los ingredientes activos utilizados en los programas de control del cultivo de arroz en el SSL fueron analizados en el laboratorio permetrina, deltametrina, triazofos y propanil, en tanto que, no 
Cuadro 1. Puntaje del índice de calidad de agua para el manejo de lagunas de inundación (ICA-L) y su relación con la calidad del agua para la vida acuática. Guanacaste, Costa Rica. 2008.

\begin{tabular}{|c|c|c|}
\hline $\begin{array}{c}\text { ICA-L } \\
\text { (puntaje) }\end{array}$ & Categoría & Descripción de la calidad del agua \\
\hline $86-100$ & Excelente & No presenta problemas para el ecosistema. Es adecuada para el desarrollo de todas las especies. \\
\hline $71-85$ & Buena & $\begin{array}{l}\text { Sostiene una alta biodiversidad de vida acuática. Se presentan períodos donde algún indicador } \\
\text { muestra peligros para el ecosistema. En este caso, si la situación no mejora en un período breve, } \\
\text { se empezarían a ver cambios en la composición del ecosistema. }\end{array}$ \\
\hline $51-70$ & Regular & $\begin{array}{l}\text { Existen signos de contaminación, como aumento en la concentración de nutrimentos. Se observa } \\
\text { una reducción de la diversidad de organismos acuáticos y un desequilibrio en el crecimiento de las } \\
\text { algas y vegetación acuática. }\end{array}$ \\
\hline $26-50$ & Mala & $\begin{array}{l}\text { Sostiene una baja biodiversidad de vida acuática, principalmente de especies tolerantes. Manifiesta } \\
\text { problemas con fuentes de contaminación puntual y no puntual. }\end{array}$ \\
\hline $0-25$ & Pésima & $\begin{array}{l}\text { Posibilita el crecimiento elevado de poblaciones de un limitado número de organismos resistentes } \\
\text { a aguas muy contaminadas. }\end{array}$ \\
\hline
\end{tabular}

Fuente: Pérez (2008).

fueron cuantificados: bispiribac-Na, acefato, butaclor, carbendazina, clomazone, esfenvalerato, fenitrotion, glifosato, imidacloprid, oxicloruro de cobre, tetraconazol y triclopyr, por implicar metodologías específicas de análisis con un costo adicional o no formar parte de los analitos de los métodos de análisis validados.

En vista de estas limitaciones y las restricciones en la frecuencia del muestreo, se evaluó el riesgo de los dieciseis plaguicidas utilizados en la plantación de arroz por medio del modelo Pesticide Index Rating Impact (PIRI) (Kookana et al. 2005), considerando su toxicidad y movilidad, con el objetivo de determinar el nivel de riesgo de contaminar tanto la LMR como el arrozal en sí, humedal artificial con presencia de aves acuáticas durante todo el ciclo de producción.

El modelo PIRI se corrió bajo las variables propias del sistema productivo y del ecosistema en estudio. Estas se recopilaron según se detalla a continuación.

\section{Variables químicas, toxicológicas y de manejo de los plaguicidas}

La etiqueta de cada producto aportó su concentración y formulación.

El programa de seguimiento al cultivo, brindó la dosis aplicada y la frecuencia. En todos los casos, las aplicaciones se hicieron al follaje y se estimó que el área tratada fue de $100 \%$.

El IRET (1999) suministró la información sobre la toxicidad del producto y el coeficiente de partición de carbono orgánico de cada plaguicida.

\section{Variables físicas}

De las visitas de seguimiento al cultivo, registros y consulta al propietario fueron cuantificadas las variables físicas: uso de suelo, área sembrada, tipo de cobertura, condiciones de humedad, período de interés, profundidad de la tabla de agua y caudal de riego que ingresó a los arrozales.

La textura y materia orgánica del suelo se obtuvieron de una muestra compuesta, formada a partir de las submuestras tomadas en cada vértice de un recorrido en zigzag que abarcó todo el sector colindante con la zona no cultivada que bordeaba la LMR. El análisis de la muestra se realizó en el Laboratorio de Suelos del Centro de Investigaciones Agronómicas de la Universidad de Costa Rica.

La distancia del borde del cultivo al cuerpo de agua, la pendiente, el ancho de la zona amortiguadora y el diámetro del cuerpo de agua fueron medidos por medio de un Sistema de Posicionamiento Global (GPS). 
Los datos de precipitación y temperatura mínima y máxima se obtuvieron de la Estación Climática de la Organización para Estudios Tropicales ${ }^{7}$.

La pérdida de suelo fue estimada a partir de la concentración promedio de sólidos suspendidos, medida en muestras del canal de desagüe del arrozal, por el Laboratorio de Calidad de Aguas de la Universidad de Costa Rica, y de los registros de caudal recibido por las parcelas durante el llenado de las terrazas, facilitados por el propietario.

\section{RESULTADOS Y DISCUSIÓN}

\section{Descripción de las prácticas agronómicas y la tec- nología utilizadas en el cultivo de arroz en el sector San Lázaro}

Aunque el área potencial de siembra de arroz en el SSL es de 1150 ha, durante la temporada agrícola del 2009 el cultivo se limitó a 350 ha bajo la modalidad de secano favorecido con riego suplementario. Para implementar el riego, el río San Lázaro, afluente natural de la LMR, fue desviado y se construyeron canales de conducción y distribución del agua y terrazas. El excedente de agua de las terrazas drenó hacia una zona pantanosa ubicada entre el arrozal y el espejo de agua de la LMR por medio de canales. Los residuos de la cosecha anterior fueron utilizados en la confección de pacas para la alimentación del ganado.

\section{Preparación del terreno y siembra}

En abril de 2009, se realizó una primera rastreada general que incorporó la cepa del arroz al suelo y en junio, donde las condiciones del suelo lo permitieron, una segunda pasada de rastra. Mientras que en áreas más bajas y con suelos más pesados, se realizó una aplicación aérea de glifosato.

El período de siembra fue entre el 20 julio y el 15 agosto de 2009, se utilizaron $100 \mathrm{~kg}$ semilla/ha de las variedades Senumisa 238, Senumisa 250, Senumisa 48 y CR-4477.

\footnotetext{
OET (ORGANIZACIÓN PARA ESTUDIOS TROPICALES). 2009. Datos Meteorológicos 1978-2009 obtenidos de la Base de Datos de Estación Biológica de la OET. Guanacaste, Costa Rica. Comunicación personal.
}

El sistema de siembra varió según las condiciones del suelo. En suelos ligeramente altos y con condiciones normales de humedad, se utilizó la máquina sembradora, en áreas con suelos pesados y con poca humedad, se empleó la máquina voleadora y en lotes con suelos muy pesados y húmedos se usó avioneta y semilla pregerminada.

Si bien, la labranza mínima mediante sembradora mecánica, o bien, al voleo con semilla pregerminada, atenuaron la posibilidad de aporte de sedimentos, el desvío de la totalidad del caudal del río San Lázaro para el llenado de las terrazas, aún cuando la precipitación fue normal, ocasionó un desperdicio considerable de agua y un aumento en el riesgo de vertido de partículas de suelo.

\section{Fertilización}

En la fertilización se utilizaron niveles de nitrógeno, fósforo, potasio, zinc y azufre considerados como promedio a los recomendados por Tinoco y Acuña (2008).

A la siembra se aplicó una dosis general de 200 $\mathrm{kg} / \mathrm{ha}$ del fertilizante $10-30-10-\mathrm{S}$. A los 15 y a los 21 días después de la germinación, se realizó la segunda fertilización con fórmulas altas en nitrógeno y potasio como la 30-0-15, a razón de $200 \mathrm{~kg} / \mathrm{ha}$.

La tercera fertilización se efectuó a los 42 días después de la siembra con la fórmula 30-0-15 a razón de $200 \mathrm{~kg} / \mathrm{ha}$, en tanto la última, se realizó a los 60-65 días con la fórmula 30-0-15 a razón de $150 \mathrm{~kg} / \mathrm{ha}$.

$\mathrm{Al}$ drenar el exceso de agua de las terrazas hacia la zona de amortiguamiento, se presume que se arrastraron fósforo y eventualmente otros potenciales contaminantes hacia LMR. Esta hipótesis estaría en consonancia con los puntajes de calidad del agua que reflejan como mayores problemas de contaminación en la salida del drenaje principal del SSL el aumento de la concentración de fósforo total, el aumento en la demanda bioquímica de oxígeno y la concentración de sólidos suspendidos.

\section{Control de enemigos naturales}

En ciertas áreas fueron aplicados los herbicidas preemergentes clomazone, triclopyr y butaclor, y en otros casos, herbicidas post-emergentes entre los 15-21 días de la germinación como bispiribac-Na y propanil.

$\mathrm{El}$ arroz fue muy sensible al ataque de gusanos defoliadores como Spodoptera sp. y Mocis sp. Para su combate se hicieron dos aplicaciones de deltametrina + esfenvalerato y imidacloprid, respectivamente. 
A los 32 días después de la siembra hubo una infestación elevada de ácaros (Steneotarsonemus spinki) y de Sogata (Tagosodes sp), y se utilizaron los insecticidas triazofos + permetrina, fenitrotion y acefato.

Fue necesaria la aplicación de oxicloruro de cobre a los 60-65 días, para el combate y prevención de bacterias (Pseudomonas sp y Xanthomonas sp).

A los 70 días cuando empezó a desarrollar y a madurar la espiga, se aplicaron fungicidas del grupo de los triasoles y la carbendazina para el control de Pyricularia grisea y Helmintosporosis (Bipolaris oryzae). En este período se combatió el chinche de la espiga ( $T i$ braca limbativentris) con deltametrina + esfenvalerato.

Un resumen de las características de los plaguicidas encontrados en uso, se presenta en el Cuadro 2.

Durante la temporada se evitó el uso de avionetas fumigadoras. La aplicación de los plaguicidas, en la mayoría del área de siembra, se hizo con helicóptero a una altura promedio de 2,0 m entre la barra de aplicación y el cultivo. En pequeñas áreas donde la saturación de los suelos posibilitó el uso del tractor, las aplicaciones de plaguicidas se hicieron con equipo terrestre. En estos casos la barra de aplicación se mantuvo a no más de 0,5 $\mathrm{m}$ respecto al cultivo. En todos los casos la aplicación fue preferiblemente antes de las 7:00 am.

Los envases de plaguicidas que se utilizaron en la temporada agrícola fueron devueltos a las casas comerciales que los distribuyen. El lavado del equipo de fumigación se realizó en un área específica, lejos de fuentes de agua superficiales.

En las muestras analizadas en el laboratorio no se logró detectar la presencia de los plaguicidas aplicados permetrina (límite de detección (LD) 0,063 $\mu \mathrm{g} / \mathrm{l}$ ), deltametrina (LD $0,039 \mu \mathrm{g} / \mathrm{l}$ ), triazofos (LD 9,7 $\mu \mathrm{g} / \mathrm{l})$ y propanil (LD $0,35 \mu \mathrm{g} / \mathrm{l})$, durante el período de muestreo de agosto a noviembre de 2009. El hecho

Cuadro 2. Plaguicidas utilizados y sus condiciones de uso en el cultivo de arroz. San Lázaro, Nicoya, Guanacaste, Costa Rica. Junio 2009.

\begin{tabular}{|c|c|c|c|c|c|c|c|}
\hline Clase & Nombre & $\begin{array}{c}\text { Ingrediente } \\
\text { activo/gl } \mathrm{o} \mathrm{cm}^{3} / \mathrm{l}\end{array}$ & $\begin{array}{l}\text { Toxicidad CL- } \\
50 \text { trucha mg/l }\end{array}$ & Koc & Dosis/ha & $\begin{array}{l}\text { Frecuencia } \\
\text { aplicación }\end{array}$ & $\begin{array}{c}\text { Tipo de } \\
\text { formulación }\end{array}$ \\
\hline \multirow[t]{6}{*}{ Herbicida } & Propanil & 0,48 & 2,3 & 518 & 7,51 & 1 & $\mathrm{CE}$ \\
\hline & Butaclor & 0,43 & 0,52 & 700 & 3,01 & 1 & $\mathrm{CE}$ \\
\hline & Glifosato & 0,41 & 38 & 6920 & 2,01 & 1 & PS \\
\hline & Clomazone & 0,48 & 19 & 244 & 1,251 & 1 & $\mathrm{CE}$ \\
\hline & Triclopyr & 0,9 & 117 & 62 & 0,61 & 1 & $\mathrm{CE}$ \\
\hline & Bispiribac-Na & 0,4 & & 302 & 0,11 & 1 & $\mathrm{CE}$ \\
\hline \multirow[t]{7}{*}{ Insecticida } & Acefato & 0,8 & 730 & 3 & 1,01 & 1 & $\mathrm{CE}$ \\
\hline & Deltametrina & 0,025 & 0,00058 & 80000 & 0,251 & 2 & CE-PM \\
\hline & Esfenvalerato & & $0,26 \mathrm{ug} / \mathrm{l}$ & 5273 & 0,251 & 1 & $\mathrm{CE}$ \\
\hline & Fenitrotion & 0,5 & 1.7 & 2000 & 0,81 & 1 & CE-PM \\
\hline & Permetrina & 0,48 & 2,5 & 20865 & 0,251 & 2 & PM \\
\hline & Imidacloprid & 0,2 & 121 & 262 & $0,25 \mathrm{~kg}$ & 1 & SC \\
\hline & Triazofos & 0,40 & 5,6 & 358 & 0,251 & 1 & CE-PM \\
\hline \multirow[t]{3}{*}{ Fungicida } & $\begin{array}{l}\text { Oxicloruro de } \\
\text { cobre }\end{array}$ & 0,84 & 2,2 & 260 & $0,5 \mathrm{~kg}$ & 2 & PM \\
\hline & Carbendazim & 0,50 & 0,83 & 400 & $0,75 \mathrm{~kg}$ & 1 & PM \\
\hline & Tetraconazol & 0,40 & 4,8 & 1152 & 0,81 & 1 & $\mathrm{SC}$ \\
\hline
\end{tabular}

Koc: coeficiente de partición de carbono orgánico; PS: polvo soluble; SC: disolución concentrada; PM: polvo mojado; CE: concentrado emulsificable. 
de que no se detectaran plaguicidas en las aguas de drenaje y en la LMR podría deberse a varios factores; entre ellos la aplicación de los plaguicidas antes de las 7:00 am en forma localizada mediante helicópteros con barras de aplicación o el uso de tractor, que pudo haber permitido mejorar la ecoeficiencia de las aplicaciones en este sistema productivo. La hora de la aplicación, cuando la intensidad del viento es mínima y el aumento en la precisión pudieron disminuir la deriva de los plaguicidas y el riesgo de contaminación del recurso hídrico vinculado al RNVS-MR.

Otro factor es la posible degradación o pérdida por volatilidad, escorrentía o adsorción de los plaguicidas, al no coincidir las aplicaciones de estos con la fecha de muestreo debido a cambios no previstos en la planificación de las actividades agrícolas. Además, el muestreo realizado de tipo puntual podría no reflejar las concentraciones promedio que tendrían las aguas a lo largo del ciclo de manejo del cultivo.

Por otra parte, debido a limitaciones presupuestarias no se incorporaron análisis químicos para detectar plaguicidas en los sedimentos de la zona comprendida entre el arrozal y el espejo de agua de la LMR, denominada zona de amortiguamiento. Lo anterior, hubiese permitido cuantificar la función de las arcillas y de las plantas acuáticas en el proceso de adsorción de los plaguicidas y otros contaminantes.

\section{Cosecha}

La cosecha se realizó entre los 100 y los 130 días después de la siembra, dependiendo de la variedad. Los rendimientos obtenidos fueron en promedio 4,46 t/ha.

\section{Calidad del agua en la laguna Mata Redonda y en las aguas de drenaje del cultivo de arroz. Sector San Lázaro}

Los valores de cada una de las variables fisicoquímicas medidas para la obtención del ICAL, en los diferentes sitios y fechas de muestreo y su respectiva calificación en relación con su calidad para el sostenimiento de la biodiversidad en las lagunas de inundación, se resumen en los Cuadros 3 y 4 , respectivamente.

Las concentraciones de fósforo (Cuadro 3 ) en la mayoría de los muestreos exceden de $0,05 \mathrm{mg} / 1 \mathrm{PO}_{4}-\mathrm{P}$,

Cuadro 3. Variables fisicoquímicas de las aguas provenientes de los arrozales del sector San Lázaro (SSL) y de la Laguna Mata Redonda (LMR) medidas durante el II semestre de 2009. Nicoya, Guanacaste, Costa Rica.

\begin{tabular}{lccccccccc}
\hline Sitio & Fecha & $\mathbf{T}^{\circ} \mathbf{C}^{*}$ & Sat $\mathbf{O}_{\mathbf{2}}$ & $\mathbf{p H}$ & $\begin{array}{c}\mathbf{D B O} \\
\mathbf{m g} / \mathbf{l}\end{array}$ & $\begin{array}{c}\mathbf{N O} \\
\mathbf{m g} / \mathbf{l}\end{array}$ & $\begin{array}{c}\mathbf{P T} \\
\mathbf{m g} / \mathbf{l}\end{array}$ & $\begin{array}{c}\text { SS } \\
\mathbf{m g} / \mathbf{l}\end{array}$ & $\begin{array}{c}\mathbf{C E} \\
\boldsymbol{\mu S} / \mathbf{c m}\end{array}$ \\
\hline Salida & $12 / 08 / 2009$ & 35,3 & 108,0 & 8,81 & 18,06 & $\leq 0,29$ & 0,2530 & 90,0 & 280,0 \\
drenaje & $22 / 09 / 2009$ & 33,6 & 80,6 & 7,17 & 6,02 & $\leq 0,29$ & 0,1349 & 57,0 & 280,0 \\
principal & $27 / 10 / 2009$ & 32,1 & 12,0 & 7,83 & 8,01 & $\leq 0,29$ & 0,1747 & 32,0 & 134,2 \\
del SSL & $24 / 11 / 2009$ & 30,8 & 135,0 & 8,05 & 6,88 & $\leq 0,29$ & 0,1772 & 44,0 & 181,1 \\
\hline Zona & $12 / 08 / 2009$ & 27,1 & 34,50 & 7,44 & 2,88 & $\leq 0,29$ & 0,1176 & 36,0 & 400,0 \\
central de & $22 / 09 / 2009$ & 27,1 & 15,20 & 7,02 & 5,38 & $\leq 0,29$ & 0,0474 & 41,0 & 250,0 \\
la LMR & $27 / 10 / 2009$ & 27,3 & 7,05 & 7,30 & 9,03 & $\leq 0,29$ & 0,0291 & 13,0 & 138,8 \\
& $24 / 11 / 2009$ & 26,7 & 20,0 & 8,07 & 4,06 & $\leq 0,29$ & 0,0565 & 21,0 & 280,0 \\
\hline Efluente & $12 / 08 / / 2009$ & 28,5 & 84,4 & 7,86 & 3,18 & $\leq 0,29$ & 0,1067 & 68,0 & 420,0 \\
principal & $22 / 09 / 2009$ & 29,2 & 29,7 & 7,21 & 4,82 & $\leq 0,29$ & $\leq 0,012$ & 31,0 & 280,0 \\
de LMR & $27 / 10 / 2009$ & 28,1 & 15,0 & 7,47 & 4,19 & $\leq 0,29$ & 0,0700 & 17,0 & 130,8 \\
& $24 / 11 / / 2009$ & 27,5 & 30,0 & 7,80 & 9,96 & $\leq 0,29$ & 0,0632 & 16,0 & 291,1 \\
\hline
\end{tabular}

* T: temperatura. Sat $\mathrm{O}_{2}$ : porcentaje de saturación de oxígeno disuelto. DBO: demanda bioquímica de oxígeno. $\mathrm{NO}_{3}$ : nitratos. PT: fósforo total. SS: sólidos suspendidos. CE: conductividad eléctrica. 
Cuadro 4. Calificación de las variables fisicoquímicas de acuerdo con el índice de calidad de agua para el manejo de lagunas de inundación (ICA-L) para el sostenimiento de la biodiversidad. Laguna Mata Redonda (LMR) y San Lázaro (SSL), Nicoya. Guanacaste, Costa Rica. 2009.

\begin{tabular}{lcccccccccc}
\hline Sitio & Fecha & $\mathbf{Q}_{\mathrm{T}}$ & $\mathbf{Q}_{\text {sat02 }}$ & $\mathbf{Q}_{\mathrm{pH}}$ & $\mathbf{Q}_{\mathrm{DBO}}$ & $\mathbf{Q}_{\mathrm{NO3}}$ & $\mathbf{Q}_{\mathrm{PT}}$ & $\mathbf{Q}_{\mathrm{SS}}$ & $\mathbf{Q}_{\mathrm{CE}}$ & $\mathbf{I C A}-\mathbf{L}$ \\
\hline Salida & $12 / 08 / 2009$ & 32 & 95 & 47 & 15 & 100 & 1 & 52 & 98 & 34 \\
drenaje & $22 / 09 / 2009$ & 56 & 87 & 90 & 50 & 100 & 6 & 67 & 98 & 57 \\
principal & $27 / 10 / 2009$ & 73 & 6 & 87 & 40 & 100 & 1 & 74 & 100 & 29 \\
del SSL & $24 / 11 / 2009$ & 84 & 84 & 84 & 46 & 100 & 1 & 70 & 100 & 48 \\
\hline Zona & $12 / 08 / 2009$ & 97 & 100 & 91 & 68 & 100 & 14 & 58 & 88 & 69 \\
central de & $22 / 09 / 2009$ & 97 & 100 & 90 & 53 & 100 & 74 & 55 & 100 & 80 \\
la LMR & $27 / 10 / 2009$ & 97 & 100 & 91 & 37 & 100 & 96 & 96 & 100 & 87 \\
& $24 / 11 / 2009$ & 97 & 100 & 84 & 58 & 100 & 64 & 93 & 100 & 86 \\
\hline Efluente & $12 / 08 / 2009$ & 95 & 100 & 87 & 66 & 100 & 21 & 63 & 86 & 72 \\
principal & $22 / 09 / 2009$ & 93 & 100 & 90 & 56 & 100 & 100 & 90 & 98 & 90 \\
de LMR & $27 / 10 / 2009$ & 96 & 100 & 72 & 57 & 100 & 50 & 93 & 100 & 82 \\
& $24 / 11 / 2009$ & 97 & 100 & 87 & 33 & 100 & 57 & 94 & 100 & 80 \\
\hline
\end{tabular}

límite que recomienda la EPA (Murphy 2002) cuando una corriente de agua fluye hacia reservorios como la LMR y si se considera el máximo de $0,025 \mathrm{mg} / \mathrm{l}$ $\mathrm{PO}_{4}-\mathrm{P}$ señalado por la EPA para lagunas rupestres, los valores en la LMR señalan un riesgo de eutrofización antropogénica.

La reiterada presencia de fósforo en las aguas de drenaje de sistemas agrícolas y en las lagunas de inundación en la zona de la cuenca del Tempisque, informadas en este y otros estudios previos (Rodríguez 1996, Rizo-Patrón 2003, Pérez 2010) plantea la necesidad de estudios isotópicos de su dinámica ambiental y la búsqueda de mejores prácticas agrícolas.

La variación espacial del ICA-L al pasar de la salida del drenaje principal del SSL a la LMR y luego, al agua del desagüe de la laguna se observa en la Figura 1.

En el drenaje principal del sembradío de arroz, el ICA-Lp fue de 42 (Figura 1) valor correspondiente a un cuerpo de agua no apto para el sostenimiento de la biodiversidad de lagunas de inundación (Cuadro 1). A este resultado contribuyen, con puntajes bajos de calidad, los residuos de fósforo, los sólidos suspendidos y los valores de DBO con puntajes bajos de calidad (Cuadro 4).

En la LMR (ICA-Lp 80) y en la salida de la laguna (ICA-Lp 81) la calidad del agua se clasificó como "buena" (Cuadro 1). Lo anterior, se relaciona con las calificaciones de la concentración de fósforo total y de DBO, variables cuyos puntajes de calidad también se vieron disminuidos en las aguas de vertido de las plantaciones del arroz del SSL (Cuadro 4).

Los puntajes de calidad para los sólidos suspendidos (Cuadro 4) indican que el arrastre de material ha llevado al drenaje principal del sembradío de arroz a mantenerse en la categoría regular. Este factor podría

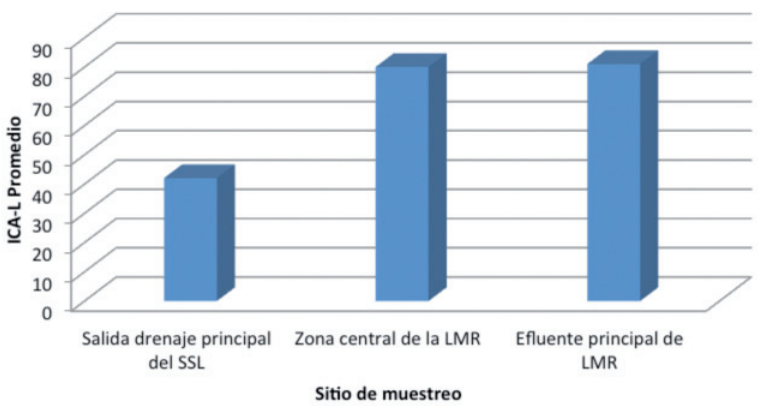

Figura 1. Clasificación de la calidad del agua por medio del índice de calidad de agua para el manejo de lagunas de inundación (ICA-L) promedio (ICALp), desde el vertido del arrozal hasta la salida del humedal. San Lázaro, Nicoya, Guanacaste, Costa Rica. 2009.

SSL: Sector San Lázaro. LMR: Laguna Mata Redonda. 
asociarse en ciertas fechas a la disminución del puntaje de calidad del fósforo total, que podría ingresar por las pérdidas de suelo y cuyos valores extremos (1 a 6), colocan a dicho cuerpo de agua en la categoría pésima e indican que estaría expuesto a un proceso de eutrofización. En contraste, el puntaje de calidad del fósforo de la zona central de la LMR y del efluente principal de LMR, varía desde pésimo a excelente en el período de estudio.

Además, los ingresos de materia orgánica biodegradable son más acentuados en el drenaje principal del sembradío de arroz, pues los puntajes de calidad de DBO (Cuadro 4) se fijan entre pésimo a malo, a diferencia de los otros dos sitios donde la categoría varía entre mala y regular.

La calidad del agua de la salida del drenaje principal del SSL se mantuvo a lo largo del período, entre la categoría regular y mala (Figura 2). En tanto que en la LMR y en el efluente principal de esta, las calificaciones se mantienen similares dentro del ámbito de la categoría buena.

La categoría buena asignada a la LMR con base en el ICA-L (Figura 1), significa según Pérez (2008), que se presentan períodos donde algún indicador muestra peligros para el ecosistema. En este caso, si la situación no mejora en un período breve, se empezarían a ver cambios en la composición del ecosistema.

Piedra (2005) informó una disminución de las poblaciones nativas de peces en la LMR y un incremento

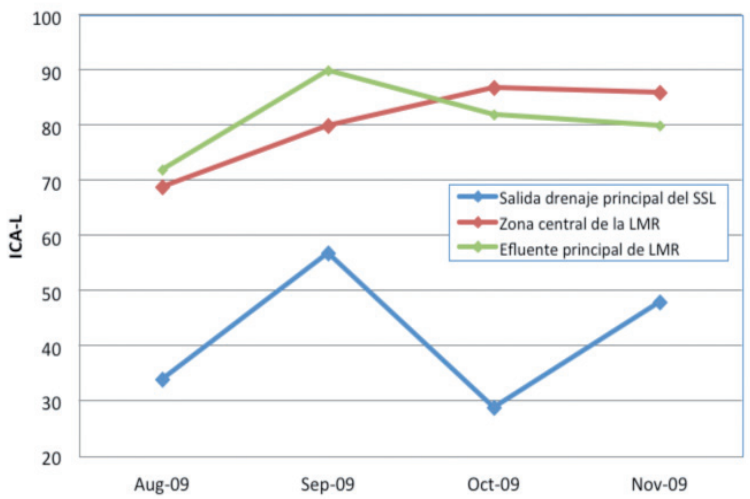

Figura 2. Variación temporal del índice de calidad de agua para el manejo de lagunas de inundación (ICA-L) de la laguna Mata Redonda y del agua que drena de los arrozales del sector San Lázaro, Nicoya, Guanacaste, Costa Rica. 2009. de las especies exóticas, más tolerantes a las condiciones de eutrofización antropogénica. Por su parte Jimé$n e z^{8}$, señaló una reducción sistemática de los espejos de agua, provocados por la invasión descontrolada de especies como el gamalote (Paspadium geminatum), mata de sapo (Ipomoea carnea), lirio de agua (Eicchornia spp), la zarza (Mimosa pigra) y otras especies de plantas acuáticas en el RNVS-MR.

La tendencia a mejorar de la calidad del agua, reflejada en la variación espacial del ICA-L al pasar de la salida del drenaje principal del SSL a la LMR (Figuras 1 y 2), se pudo deber al efecto de la zona de amortiguamiento y al conocido valor de los humedales como ecosistemas para purificación del agua.

Al comparar los valores de ICA-Lp del estudio de Pérez (2010), en la Laguna La Bocana del Parque Nacional Palo Verde y los arrozales colindantes, con los de este trabajo, se observa que las salidas de los canales de drenaje se mantienen en la categoría "mala" y sus puntajes (42 para SSL y 50 para el sector de riego Tamarindo), resultan equivalentes considerando las desviaciones. Por su parte, los valores de las lagunas presentan mayor diferencia, al quedar en categorías diferentes, regular para Palo Verde y buena para LMR. Sin embargo, los puntajes de 70 y 80 , respectivamente, por la dispersión de los resultados quedarían superpuestos.

En ambos estudios las salidas de los canales de drenaje de las parcelas mantienen valores altos de fósforo, lo que hace suponer que se tendrían pérdidas de fertilizante fosfórico. Es interesante acotar que la concentración promedio del fósforo disminuye a una tercera parte al analizar el agua de la laguna rupestre. Los sólidos suspendidos, se mantuvieron en valores inferiores a los de la salida de los canales de drenaje.

\section{Riesgo ambiental de los plaguicidas utilizados en el sector de San Lázaro}

Los arrozales inundados por ser zonas de alimentación para las aves acuáticas requieren un cuidado extremo del productor, para evitar que su riqueza faunística esté expuesta a altos niveles de contaminantes, producto de las aplicaciones de plaguicidas durante el ciclo de cultivo (Rizo-Patrón 2003, Trama 2005).

8 Jiménez, A. 2009. Prevalencia de malezas en la laguna Mata Redonda RNVS-MR- ACT- SINAC. Rosario, Nicoya, Guanacaste, C.R. Comunicación personal. 
La ausencia de criterios ambientales en la selección de los plaguicidas, a pesar de su amplia gama de niveles de toxicidad, tendría un efecto especialmente nocivo en aquellos años donde las condiciones ambientales y de mercado fueran favorables para un segundo ciclo de siembra. Típicamente este se realiza en $40 \%$ del área de siembra tradicional y comprende los meses de enero, febrero y marzo. En esta época, por concentrarse grandes cantidades de aves acuáticas (migratorias y residentes), el riesgo de contaminación de las aguas por la utilización de plaguicidas tóxicos y móviles, conllevaría mayores daños de intoxicación de peces y aves acuáticas, tanto en el arrozal como en la totalidad del RNVS-MR.

La información recopilada para reunir criterios ambientales para la selección de los plaguicidas, considerando el riesgo por factores de movilidad y toxicidad, se reúne en el Cuadro 5.

En la Figura 3, originada a partir del modelo PIRI, se observa que el propanil tendría una movilidad alta

Cuadro 5. Resumen de variables de la zona de estudio sobre la calidad del agua del Refugio Mata Redonda y arrozales colindantes, aplicadas en el modelo Pesticide Index Rating Impact. San Lázaro, Nicoya, Guanacaste, Costa Rica. II semestre 2009.

\begin{tabular}{|c|c|c|}
\hline Variable & Valor utilizado & Fuente y otras observaciones \\
\hline Uso de suelo & cultivo de arroz & \\
\hline Área sembrada (ha) & 325 & Entrevista con productor \\
\hline Textura de suelo & arcillosa & Análisis de suelo \\
\hline Tipo de cobertura de suelo & follaje & \\
\hline Contenido de materia orgánica $(\%)$ & 1,6 & Análisis de suelo \\
\hline $\begin{array}{l}\text { Condiciones de humedad del suelo en el período de } \\
\text { interés }\end{array}$ & saturado & $\begin{array}{l}\text { Visita de campo y observación de humedales y de } \\
\text { cultivo de arroz inundado }\end{array}$ \\
\hline Inicio de período de interés & 15 julio 2009 & \\
\hline Final de período de interés & 30 noviembre 2009 & \\
\hline Profundidad de la tabla de agua (m) & 0,00 & Arroz inundado \\
\hline Diámetro del cuerpo de agua más cercano al cultivo (m) & 950 & $\begin{array}{l}\text { Estimado con sistema de información geográfica. } \\
\text { Setiembre } 2009\end{array}$ \\
\hline Distancia del borde del cultivo al cuerpo de agua (m) & 850 & $\begin{array}{l}\text { Estimado con sistema de información geográfica. } \\
\text { Setiembre } 2009\end{array}$ \\
\hline Pendiente del terreno al cuerpo de agua (\%) & 1,5 & Estudio topográfico Ing. Matarrita ${ }^{9}$ \\
\hline Ancho de la zona amortiguamiento (m) & 950 & $\begin{array}{l}\text { Estimado con sistema de información geográfica. } \\
\text { Setiembre } 2009\end{array}$ \\
\hline Pérdida de suelo estimada en el período de interés ( $\mathrm{t} / \mathrm{ha}$ ) & 0,2 & $\begin{array}{l}\text { Estimación a partir de registros de caudal de ingreso y } \\
\text { concentración de sólidos suspendidos }\end{array}$ \\
\hline Precipitación total en el período de interés (mm) & 1817 & $\begin{array}{l}\text { Datos de estación meteorológica de OET en PNPV } \\
\text { (OET 2009) }\end{array}$ \\
\hline Irrigación total en el período de interés. $\left(\mathrm{m}^{3}\right)$ & 691200 & $\begin{array}{l}\text { Caudal del río San Lázaro que ingresa al arrozal en } \\
\text { agosto }\end{array}$ \\
\hline & & $1,2 \mathrm{~m}^{3} / \mathrm{s}$ durante 20 días \\
\hline $\begin{array}{l}\text { Temperatura promedio mínima en el período de interés } \\
\left({ }^{\circ} \mathrm{C}\right)\end{array}$ & 25,5 & $\begin{array}{l}\text { Datos de estación meteorológica de OET en PNPV } \\
\text { (OET 2009) }\end{array}$ \\
\hline $\begin{array}{l}\text { Temperatura promedio máxima en el período de interés } \\
\left({ }^{\circ} \mathrm{C}\right)\end{array}$ & 28,3 & $\begin{array}{l}\text { Datos de estación meteorológica de OET en PNPV } \\
\text { (OET 2009) }\end{array}$ \\
\hline Mínimo de días entre la aplicación y la lluvia o riego & 1 & Condiciones climáticas usuales \\
\hline
\end{tabular}

Fuente: Matarrita (2012).

9 Matarrita, J. 2009. Estudio de niveles en RNVS-MR. (Entrevista). Guanacaste, CR, SENARA. 


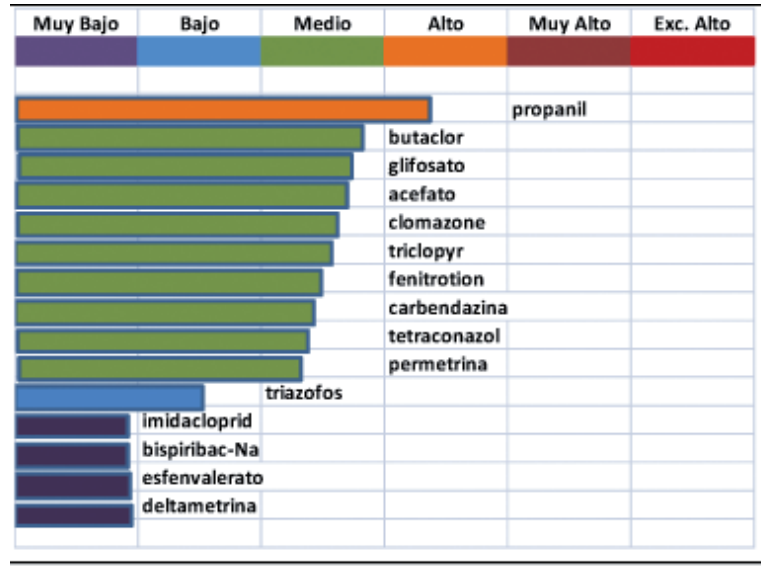

Figura 3. Movilidad estimada de los plaguicidas en agua superficial, bajo las condiciones específicas del cultivo de arroz en el sector San Lázaro, según el modelo Pesticide Index Rating Impact. Nicoya, Guanaste, Costa Rica. 2009.

en el agua superficial en el SSL, mientras que para los plaguicidas butaclor, glifosato, acefato, clomazone, triclopyr, fenitrotion, carbendazina, tetraconazol y la permetrina, el modelo predice una movilidad media. Finalmente los plaguicidas con movilidad baja o muy baja, corresponden a triazofos, imidacloprid, bispiridac-Na, esfenvalerato y deltametrina.

En el SSL, el uso de productos con movilidad alta como el propanil (Figura 3) aumenta el riesgo de su traslado en el agua de escorrentía. Esta característica advierte sobre su mayor potencial de convertirse en causa de contaminación de la LMR, especialmente si se aplica una alta dosis de producto, no se controla el volumen de agua y se reduce la zona de amortiguamiento por presiones económicas.

Otros productos con movilidad media (Figura 3) mantienen el riesgo de contaminar la LMR y su uso debería condicionarse tomando en cuenta su grado de toxicidad y la forma de aplicación para evitar eventos de contaminación. En el caso de los productos con movilidad baja, las condiciones de aplicación que conlleven una alta deriva, por ejemplo mediante aplicaciones aéreas con avioneta, mantendrían el riesgo de contaminar la LMR.

El modelo PIRI, predijo que los insecticidas esfenvalerato y permetrina tendrían un nivel de toxicidad muy alto, bajo las condiciones del SSL, en tanto que otros siete plaguicidas del paquete tecnológico empleado tienen una toxicidad muy baja (Figura 4).

En el Cuadro 6 se presenta la matriz que combina la información sobre movilidad y toxicidad generada al aplicar el PIRI. Este cuadro permite tener un criterio ambiental para seleccionar los agroquímicos. De esta manera, el acefato, clomazone, glifosato, triclopyr, triazofos, bispiribac-Na, imidacloprid y tetraconazol, son los productos que generarían el menor impacto sobre las aguas que drenan hacia la LMR.

En el Cuadro 6 se conjuntan los riesgos de toxicidad y movilidad. La deltametrina, el butaclor, el esfenvalerato y la permetrina, presentarían un alto riesgo de producir daños en la biodiversidad asociada al cultivo del arroz por su alta toxicidad (Figura 4), pero muy poco riesgo de producir daños en la LMR bajo condiciones de aplicación adecuadas, debido a su movilidad de baja a media.

La movilidad y el nivel de toxicidad estimados con la aplicación del PIRI, bajo las condiciones del SSL, dan un criterio para seleccionar los plaguicidas más adecuados si se considera la protección de la LMR. No obstante, como el cultivo de arroz es un humedal donde es común encontrar aves acuáticas y otros organismos alimentándose (Hurtado 2003), la movilidad deja de ser relevante, pues basta una alta toxicidad para aumentar el riesgo de dañar la biodiversidad en el sitio de aplicación (Cuadro 6).

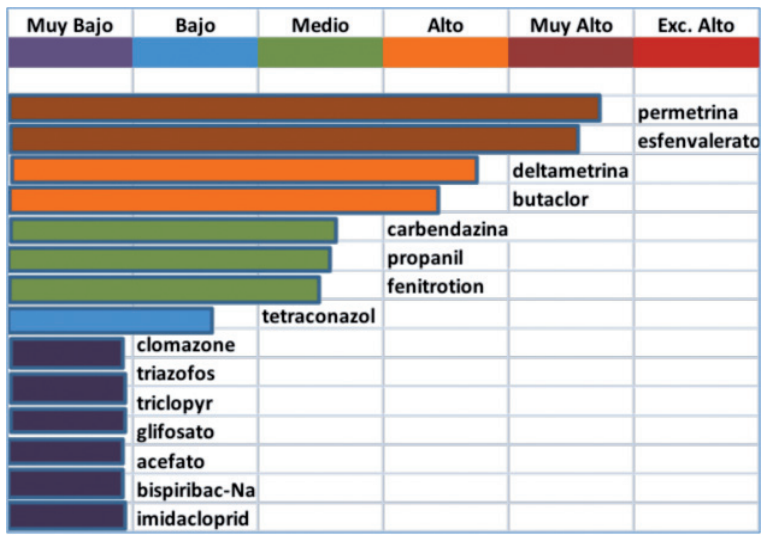

Figura 4. Riesgo por toxicidad estimado para los plaguicidas aplicados en el cultivo de arroz bajo las condiciones del sector San Lázaro, según el modelo PIRI. Nicoya, Guanaste, Costa Rica. 2009. 
Cuadro 6. Matriz de la movilidad contra la toxicidad de los plaguicidas utilizados para el cultivo de arroz en el sector San Lázaro estimada, según el modelo PIRI, Nicoya, Guanacaste, Costa Rica. 2009.

\begin{tabular}{|c|c|c|c|c|c|}
\hline $\begin{array}{l}\text { Toxicidad } \\
\text { Movilidad }\end{array}$ & Muy baja & Baja & Media & Alta & Muy alta \\
\hline \multicolumn{6}{|l|}{ Muy alta } \\
\hline Alta & & & Propanil & & \\
\hline Media & $\begin{array}{c}\text { Acefato } \\
\text { Clomazone } \\
\text { Glifosato } \\
\text { Triclopyr }\end{array}$ & Tetraconazol & $\begin{array}{l}\text { Carbendazina } \\
\text { Fenitrotion }\end{array}$ & Butaclor & Permetrina \\
\hline Baja & Triazofos & & & & Esfenvalerato \\
\hline Muy baja & $\begin{array}{l}\text { Bispiribac- Na } \\
\text { Imidacloprid }\end{array}$ & & & Deltametrina & \\
\hline
\end{tabular}

El fenitrotion, la carbendazina y el propanil con una predicción de toxicidad media (Figura 4) y una movilidad entre media y alta, no serían los productos a escoger como primera opción y deberían ser manejados asegurando buenas prácticas agrícolas, pues podrían repercutir negativamente en los organismos asociados al cultivo de arroz y al RNVS-MR.

Los plaguicidas imidacloprid, bispiribac-Na, triazofos, glifosato, acefato, clomazone, tricopyr y el tetraconazol, de acuerdo con la evaluación del PIRI, tienen una toxicidad de muy baja a baja (Figura 4) y una movilidad entre baja y media. Por lo tanto, presentan características para ser incorporados en un programa de Buenas Prácticas Agrícolas para el cultivo de arroz del SSL. Lo anterior, porque su riesgo potencial para afectar a la biodiversidad asociada al cultivo de arroz y a la LMR bajo condiciones adecuadas de aplicación es reducido.

\section{AGRADECIMIENTOS}

Al Área de Conservación Tempisque del Sistema Nacional de Áreas de Conservación, por su apoyo al desarrollo de la investigación y a la Fundación para el equilibrio entre la conservación y el desarrollo (FUNDECODES) por el financiamiento aportado a este proyecto. Al personal del Refugio Nacional de Vida Silvestre Mata Redonda, especialmente a Biol. Andrés
Jiménez Solera, señores Gabino Carrillo Arias, Martín Pérez Hernández y Jacinto Carrillo Reyes, por su colaboración en el trabajo de campo.

\section{LITERATURA CITADA}

Di Corcia, A; Marchetti, M. 1992. Method development for monitoring pesticides in environmental waters. Liquidsolid extraction followed by liquid chromatography. Environ. Sci. Technol 26(1):66-67.

Di Corcia, A; Samperi, R. 1993. Graphitized carbon black extraction cartridges for monitoring polar pesticides in water. Anal. Chem. 65(7):902-912.

Eaton, AD; Clesceri, LS; Rice, EW; Greenberg, AE. 2005. Standard methods for the examination of water and wastewater. 2 ed. Washington, DC, USA, APHAAWWA-WEF. p. 1.27-1.35, 2.38, 4.3-4.5, 4.152.

FDA (Food and Drug Administration). 1999. Pesticide analytical manual. Volume I: Multiresidue methods. 3 ed. USA, US Department of Health and Human Services, Public Health Services, Food and Drug Administration. 94 p.

Hurtado, J. 2003. Abundancia, diversidad, riqueza, uso de hábitat y comportamiento de aves acuáticas: una comparación entre un humedal seminatural y un arrozal con riego en Costa Rica. Tesis Maestría. Heredia, Costa Rica, Universidad Nacional. 43 p. 
IRET (Instituto Regional de Estudios de Sustancias Tóxicas) 1999. Manual de plaguicidas: guía para América Central. 2 ed. Heredia, Costa Rica, Universidad Nacional. p. 1-118.

Jiménez, J; González, E. 2001. La Cuenca del Río Tempisque. Perspectivas para un manejo integrado. Organización para Estudios Tropicales. San José, Costa Rica. 150 p.

Jiménez, J; González E. 2003. Hacia el manejo integral de la cuenca baja del río Tempisque. El Desafío de una región. San José, Costa Rica, Organización de Estudios Tropicales. $12 \mathrm{p}$.

Kookana, R; Correll, R; Miller, R. 2005. Pesticide impact rating index-A pesticides risk indicator for water quality. Water, Air and Soil Pollution Focus 5:45-46.

Matarrita, JF. 2012. Evaluación de la influencia de la actividad arrocera sobre la calidad del agua que drena hacia el Refugio Nacional de vida silvestre Mata redonda, Nicoya, Guanacaste, Costa Rica. San José, C.R., Universidad de Costa Rica, Facultad de Ciencias Agroalimentarias, Escuela de Agronomía. 105 p.

Murphy, S. 2002. Research analyst, BASIN project, City of Boulder. USGS water quality monitoring (en línea). Consultado 10 ene. 2012. Disponible en bcn.boulder. co.us/basin/watershed/wqhome.html

Pérez, AG. 2008. Índice físico químico de la calidad del agua para el manejo de lagunas tropicales de inundación. Revista Biología Tropical 56(04):1905-1918.
Pérez, AG. 2010. Evaluación de las aguas de drenaje del sector de riego de Tamarindo y su influencia sobre el Parque Nacional Palo Verde. Ciencia y Tecnología 26(1 y 2):71-86.

Piedra, L. 2005. Poblaciones ícticas del humedal Mata Redonda: el manejo para su conservación. Informe final. San José, Costa Rica, INBIO-MINAE. 28 p.

Rizo-Patrón, F. 2003. Plaguicidas, nutrientes y sólidos suspendidos en un humedal de Costa Rica. Tesis de Maestría, Heredia, Costa Rica, Universidad Nacional. $120 \mathrm{p}$.

Rodríguez, A. 1996. Informe final "Vigilancia de la calidad de las aguas superficiales en la zona de influencia del Proyecto de Riego Arenal Tempisque". CICA, Universidad de Costa Rica, Costa Rica. 38 p.

Tinoco, R; Acuña, A. 2008. Cultivo de arroz (Oryza sativa). Manual de recomendaciones técnicas. San José, Costa Rica, Instituto de Innovación y Transferencia de Tecnología Agropecuaria. 74 p.

Trama, F. 2005. Manejo activo y restauración del humedal Palo Verde: cambios en la cobertura de vegetación y respuestas de las aves acuáticas. Tesis de Maestría. Heredia, Costa Rica, Universidad Nacional. p. 54-78.

Valverde, JM. 2006. Mapa: zona de amortiguamiento del Refugio Nacional de Vida Silvestre-Mata Redonda. 1 ed. Hojancha, Guanacaste. SIG-ACT. Esc 1:50.000. $1 \mathrm{p}$. 